\title{
IMMIGRANTS' MENTAL HEALTH SERVICE USE COMPARED TO THAT OF NATIVE FINNS: A REGISTER STUDY
}

\author{
Valentina Kieseppä*, Minna Torniainen-Holm, Markus Jokela, Jaana Suvisaari, Mika Gissler, Niina Markkula and \\ Venla Lehti \\ *National Institute for Health and Welfare, valentina.kieseppa@thl.fi
}

\section{INTRODUCTION}

Many aspects related to migration may predispose immigrants to mental health problems. Studies have indicated that immigrants have a greater risk of developing some mental illnesses than native populations. Yet it has been repeatedly found that immigrants are less likely to use mental health services compared to natives. The aim of this study was to compare the intensity of mental health treatment with specialized mental health care between immigrants and native Finns. We also studied how the region and country of origin, and the length of residence in Finland affected the intensity of psychiatric treatment.

\section{MATERIAL AND METHODS}

The study participants were identified from the Central Population register of Finland. Explanatory variables of interest included length of residence in Finland, region and country of origin, sex, age and socioeconomic status. Psychiatric treatment received was measured as the number of visits in mental health services and was categorized as follows:

\section{Low intensity (1-3 visits) \\ 2. Moderate intensity (4-10 visits) \\ 3. High intensity (over 10 visits)}

Multinomial logistic regression was used to study the effect of immigrant status and other explanatory variables on the length of treatment.

\section{RESULTS}

Figure 1 shows the differences in treatment intensity between immigrants and Finnish-born controls. In the first model, we tested the interaction between immigrant status and all other covariates, but only interaction between age and immigrant status was significant (shown in Figure 2).

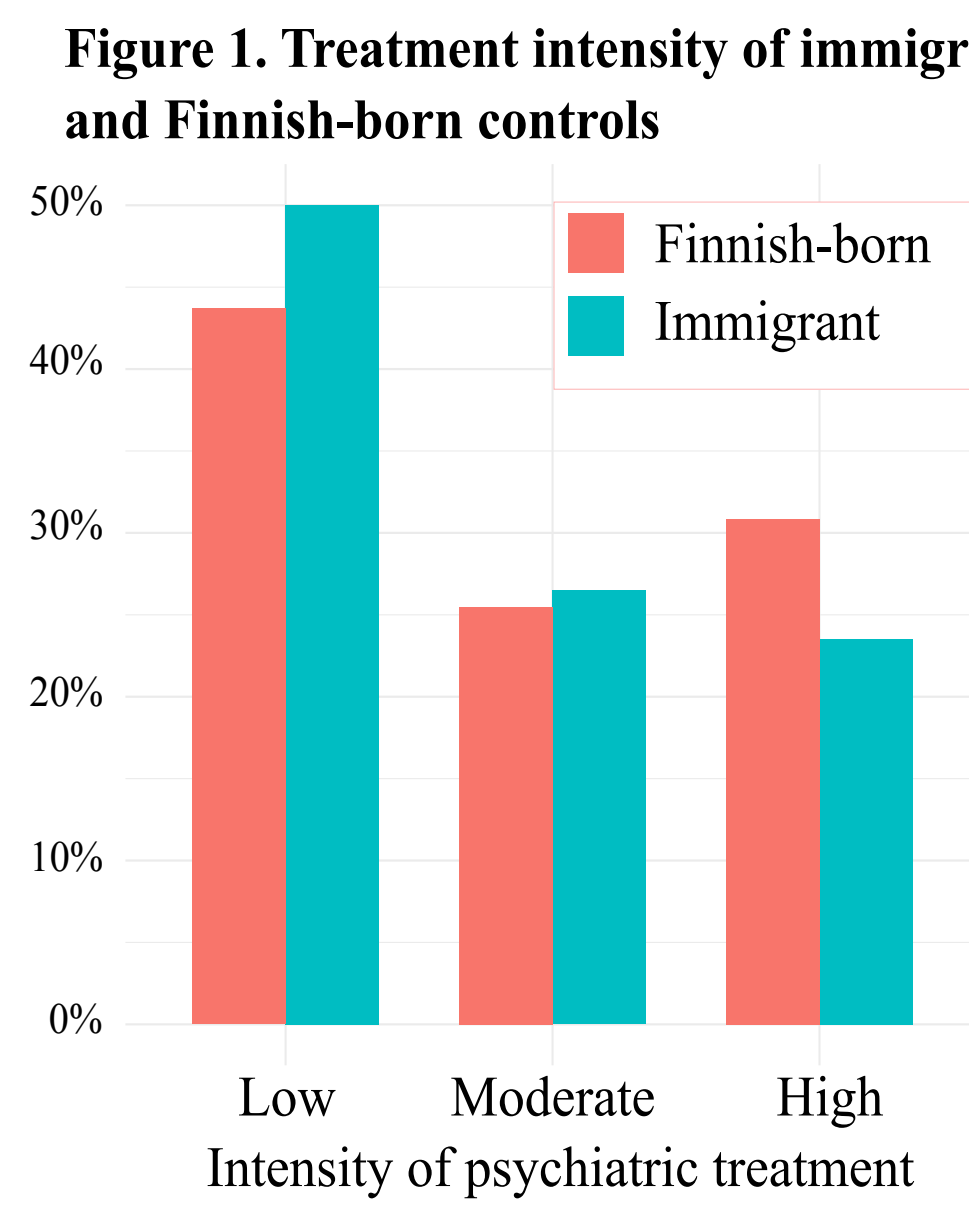

Figure 2. The interaction effect between age and immigrant status on low treatment intensity

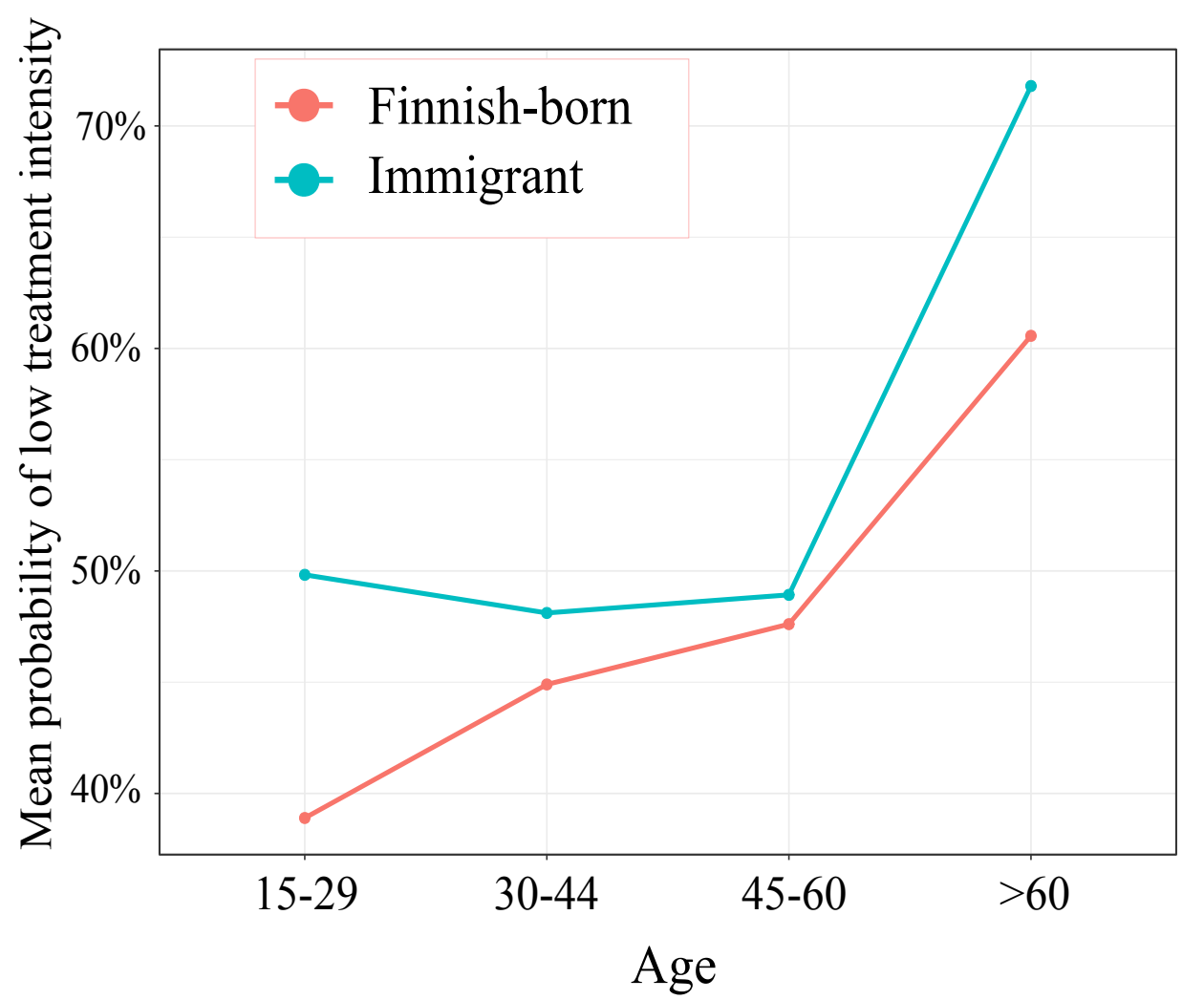

Figure 3 and 4 show odds ratios for low treatment intensity within immigrants. "High intensity" is the reference category for the outcome variable in both models (both models were adjusted for sex and age).

Figure 3. Odds ratios for low treatment intensity with length of residence and region of origin as predictors

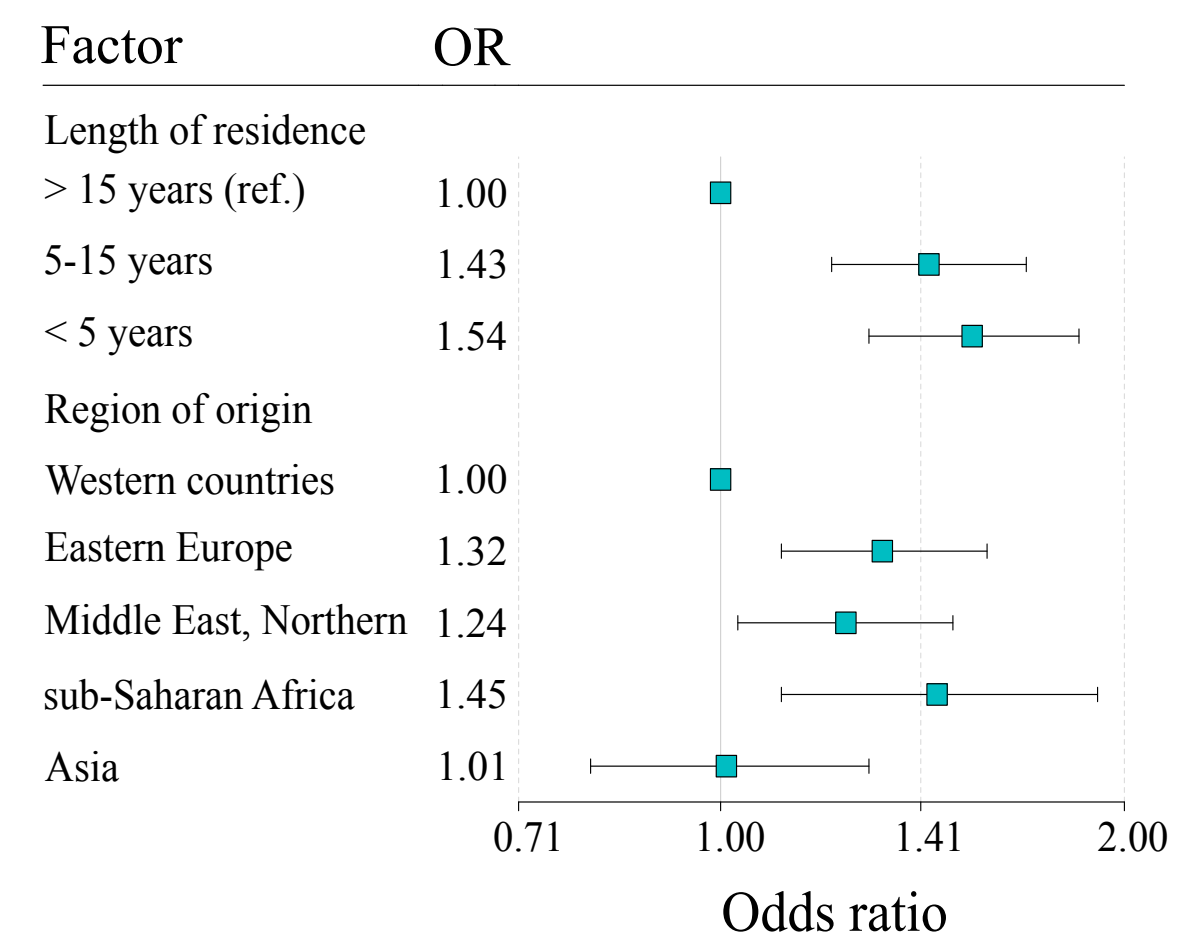

Figure 4. Odds ratios for low treatment intensity with country of origin as a predictor

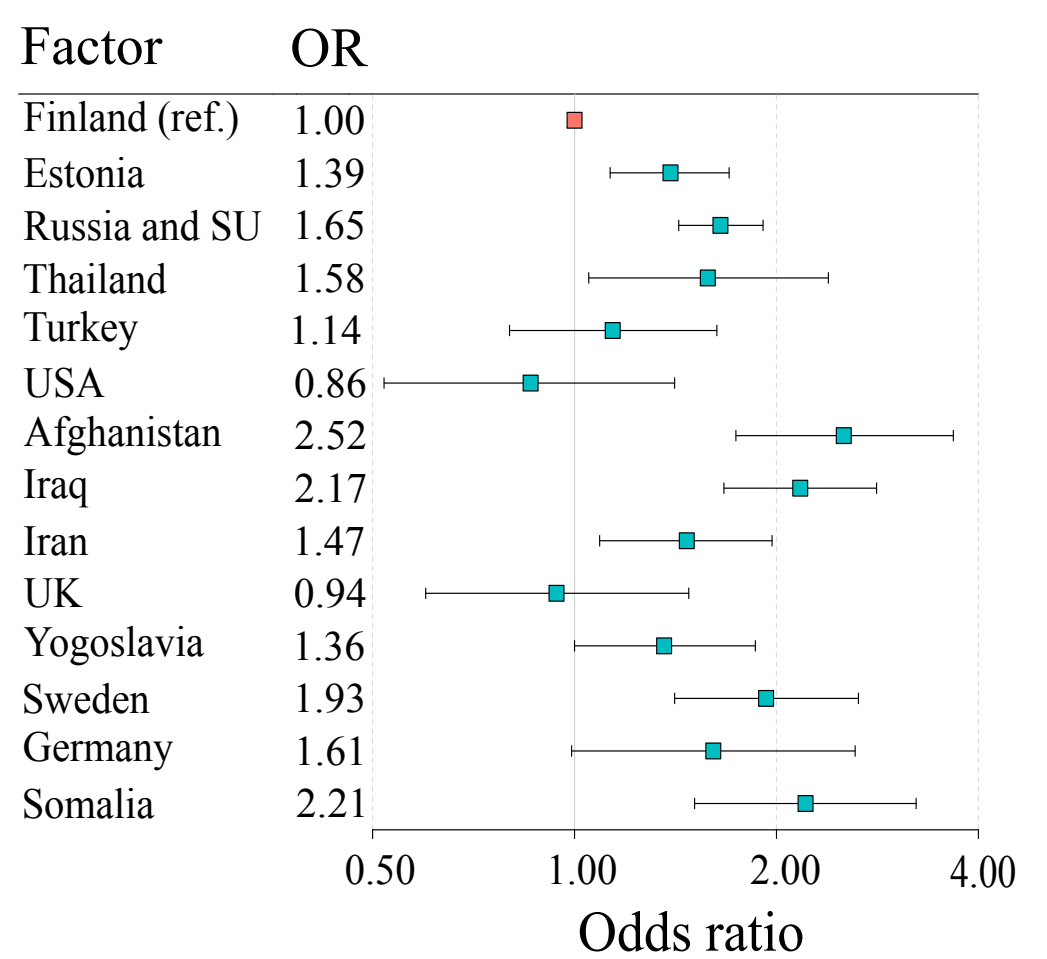

The original sample

All immigrants living in Finland aged at least 15 at the end of $2010(n=185605)$

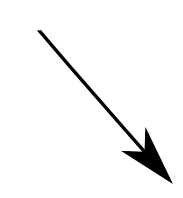

The sample used in the current study

Those who used spezialized mental health services at least once during 2011-2014 and had no contacts with pscyhiatric services in the preceding

two years (immigrants $n=6059$, controls $n=8226$ )

Matched Finnish-born controls aged at least 15 at the end of $2010 \quad(n=185605)$ \section{controls}

- Immigrants used mental health services at lower intensity compared to Finnish-born

- In comparison within immigrants, those from Eastern Europe, Middle East and Africa were especially likely to receive treatment of low intensity. Length of residence in Finland predicted higher treatment intensity.

- At highest risk for receiving low-intensity treatment were immigrants from Afghanistan, Somalia and Iraq.

- Age had different effect for immigrants and controls: for controls, the effect was linear, but among immigrants the youngest and the oldest immigrants were at highest risk for low-intensity treatment. 